\title{
Effects of Self-Esteem on Self-Face Recognition: An Eye Movement Study
}

\author{
Yuanyan $\mathrm{Hu}^{1,2}$, Shasha Liao ${ }^{1}$, Wenbo Luo ${ }^{1}$, Weijie $\mathrm{He}^{1^{*}}$ \\ ${ }^{1}$ School of Education, Chongqing University of Arts and Sciences, Chongqing, China \\ ${ }^{2}$ Laboratory of Cognition and Mental Health, Chongqing University of Arts and Sciences, Chongqing, China \\ Email: huyypsy@126.com, "hwj0130@qq.com
}

Received November 2013

\begin{abstract}
In this study, effects of self-esteem on self-faces recognition toward four types of faces were evaluated by eye movements tracking within a self-face recognition paradigm. Rosenberg's Self-Esteem Scale (RSES) was used to assess self-esteem level of participants, high $(n=16)$ and low $(n=16)$ self-esteem undergraduates participated in it. Participants completed a self-face recognition task wherein eye movements were recorded during the presentation of self-face, partner's face, famous face and stranger face. Findings showed that high self-esteem group pay more attention to self-face, partner' face and famous face than unfamiliar face; low self-esteem individuals, however, pay the same attention to all categories. These results not only enriched the research about the self-esteem, but also the self-face recognition.
\end{abstract}

Keywords: Self-Esteem; Eye Movement; Self-Face Recognition; Pupil Size

\section{Introduction}

Self-awareness is an individual's subjective perception and judgment towards his own physical, mental and social adaptation ability. It refers to one's self-consciousness which includes: self-perception, self-experience, and self-monitoring (Duval \& Wicklund, 1972). Self-face identification falls in the category of self-perception and it is one of the key indicators of an individual's level of self-awareness. Being a significant social visual stimulus, self-face is of special meaning to humans due to its strongest level of stimulation to one-self and uniqueness comparing to one's name, hence it is recognized as the most powerful and straight-forward cue in self-information processing studies (Kircher et al., 2001).

Previous studies have shown that the processing of self-relevant information differs from the processing of general information. According to Keenan et al. (1999), one's recognition towards his own face is significantly faster than to the faces of his acquaintances or strangers; In a visual search task, participants' response towards their own faces is faster than that of the unknown faces when they are exposed to a mixed faces background-this advantage remains effective even after hundreds of time's practice towards unknown faces (Tong \& Naka-yama, 1999); another experiment in examining whether the signs of familiar faces is relevant to one's angle of observation also revealed that one's self-naming is significantly earlier than that of other faces unknown (Troje \& Gersten, 1999).

As part of self-experiences embedded in the self-awareness, self-esteem is a distinctive emotional assessment one has to himself either positive or negative. Most studies of self-esteem have been focused on the thoughts and moods. For example, comparing individual with low self-esteem, those with high self-esteem have been showed to possess clearer self-concepts (Campbell \& Lavallee, 1993); to be less vulnerable to loneness

\footnotetext{
*Corresponding author.
}

and depression (Creemers et al., 2012); to persist in the face of failure (Di Paula \& Campbell, 2002), and to perceive negative feedback as a challenge rather than a threat (Seery et al., 2004). It should be noted that little empirical study on self-esteem has been focused on the self-face recognition. However, what kind of characteristics individuals with diverse levels of self-experiences, especially individuals with high self-esteem and those with low self-esteem would have in their self-face recognition, have been mentioned in literatures therefore answers to this question would be of significance in helping to explore human psychological functions, especially self-face recognition and self-awareness.

Additionally, with the development of eye tracking technology, physical measures such as the pupil size, fixation duration and fixation counts have also been used by researchers in their studies in human's information processing, which has greatly strengthened our explorations to various human psychological activities (Zhang \& Ye, 2006).

To better understand the details of information-processing on the self-face recognition across different self-esteem levels, we used pictures of oneself and others (e.g. one's partner, unfamiliar and famous people) to examine not only the behavioral response, but also eye movement data. Thus, our primary questions were the following: 1) Could we detect differences in participants behavioral response between their self-face and others' face? 2) Could we detect differences between participants of high self-esteem and low self-esteem's in their reactions during the self-face identification? 3) Did high self-esteem individuals display different eye movement from low self-esteem individuals?

\section{Method}

\section{Participants}

The final participants were 32 undergraduates recruited from 
Chongqing University of Arts and Sciences in Chongqing, China. They ranged from 17 to 22 year old $(M=19.9)$. They were paid for their participation. They all had normal uncorrected vision or their vision was corrected via glasses. All participants were Chinese and right-handed, and were required to choose a partner of his/hers from the 32 participants to match either of the gender.

\section{Measures}

The Rosenberg's Self-esteem Scale (RSES) is a widely used measure to establish participants' self-esteem level (Rosenberg, 1965). 34 participants were selected from 202 undergraduates, they were split into two groups (17 participants in each group), including high $(\geq 33)$ and low $(\leq 23)$ group.

\section{Apparatus}

Eye movements were recorded using a video-based iView X Hi-speed tracking system (SensoMotoric Instruments, Germany) with a sampling rate of $250 \mathrm{~Hz}$ connected to a Core II DELL compatible computer. All stimuli were presented on a gray background on a 21-inch monitor $(85 \mathrm{~Hz})$ with a screen resolution of $1024 \times 768$ pixels. In order to minimize the head movements and keep distance of $80 \mathrm{~cm}$ between the participants and the center of the stimulus display screen, participants were required to put their chin on a soft head restraint and keep their forehead close to the concave panel. Before the formal task, a standardized calibration procedure was performed by requiring participants to focus on 9 black dots randomly appearing on the gray display.

\section{Stimuli}

Each participant was photographed in similar conditions, the image was used as "self-face” for him/herself and as "partner's face" for his/her partner. The images of unfamiliar were obtained from the Chinese College Students' Affective Face Picture Data Base (Wang \& Luo, 2005). Morph Editor (SoftKey Corporation, Cambridge, MA) was used to create digital morphs between the subjects' and the others, resulting in 21 unique faces. All images were edited by Adobe Photoshop CS 2 in order to remove external features (hair, ears) and created on a uniform grey background (Figure 1). A scrambled face was created by randomly rearranging one image.

\section{Tasks}

There were three blocks for every participant and each block was composed of 50 trials, totally 150 trials per participant. Half of the participants were required to click the "up" key of mouse if the image was himself/herself and the "down" key for others.

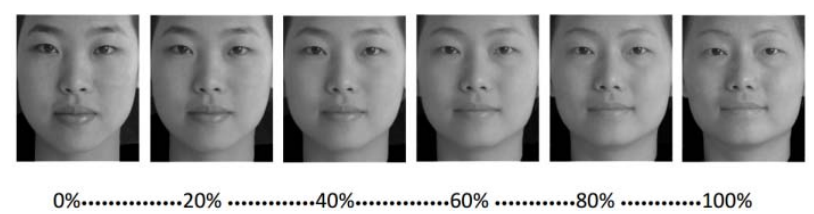

Figure 1.

Self-partner morph series.

\section{Results}

\section{Behavioral Data}

Data from 2 participants were excluded due to a loss of behavioral and eye movement integrity. A two-way repeated measures ANOVA was performed to determine whether there was a difference in recognizing the self-face, partner's face, famous face and unfamiliar face depending on whether the participant is high or low self-esteem. The main effect of image types was significant, $F(3,90)=7.262 ; p<0.001$. Post hoc comparisons revealed that, while the mean response time of self-faces (578 ms) was faster than those to unfamiliar faces (716 ms) $(p<0.01)$ and famous face $(632 \mathrm{~ms})(p<0.05)$, the mean response time was not significant between self-face and partner's face $(607 \mathrm{~ms})(p>0.05)$. However, the main effect of self-esteem was not significant, and the interaction between the self-esteem and image types was not significant either (Figure 2).

\section{Eye Movement Data}

A two-way repeated measures ANOVA with Self-esteem (high vs. low self-esteem) and Image types (self, unfamiliar, famous and partner) as factors were conducted on pupil size, fixation duration, and fixation count respectively.

For pupil size, the interaction between Self-esteem level and Image types was significant, $F(3,90)=3.771, p=0.013$. Simple effect revealed that for the high self-esteem group, the pupil size of self-face was significantly larger than unfamiliar face ( $p$ $<0.05$ ); however, the difference among self-face, partner's face, and famous face was not significant $(p>0.05)$. In contrast for the low self-esteem group, the difference among the four types of images was not significant $(p>0.05)$. The main effect of self-esteem level was not significant $F(1,30)=0.101, p=$ 0.753 , and the main effect of image types was not significant either, $F(3,90)=0.323, p=0.809$ (Table 1).

For either first fixation duration or first fixation count, the interaction between the face types and self-esteem was not significant. Neither main effect of image types nor main effect of self-esteem was significant, $p>0.05$.

\section{Discussion}

Consistent with previous studies (Keenan et al., 1999; Tong

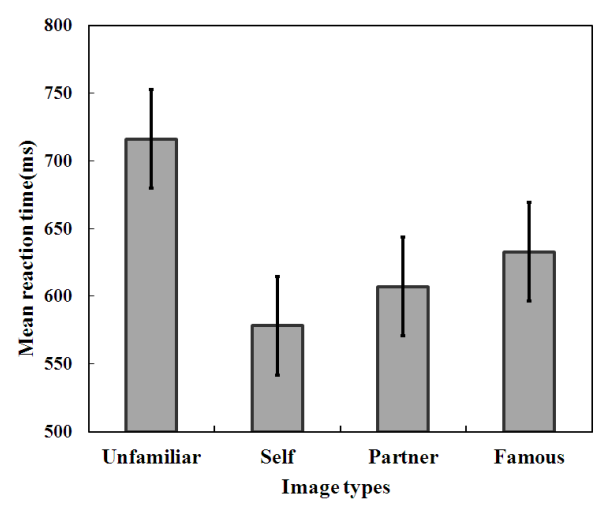

Figure 2.

Mean reaction time to different images of all participants. 
Table 1.

Mean and standard deviation of pupil size for high and low self-esteem participants.

\begin{tabular}{cccccc}
\hline \multicolumn{2}{c}{ SE level } & Self-face & Partner & Famous & Unfamiliar \\
\hline \multirow{2}{*}{ High } & $M$ & 876.05 & 784.20 & 782.07 & 773.63 \\
& $S D$ & 221.05 & 235.62 & 242.44 & 238.28 \\
\multirow{2}{*}{ Low } & $M$ & 738.66 & 798.62 & 781.56 & 799.18 \\
& $S D$ & 244.03 & 234.88 & 231.73 & 257.80 \\
\hline
\end{tabular}

\& Nakayama, 1999; Sui, Zhu, \& Han, 2006), our behavioral data indicated that responses to self-faces were faster than those to others (famous, and unfamiliar faces). It can be explained by the self-face recognition advantage effect that humans respond faster to self-faces than to other faces, and this has been demonstrated over either familiar or unfamiliar faces (Sui, Zhu, \& Han, 2006). In contrast, consistence with Kircher et al. (2001), the reaction time between self-face and partner's face was the same. The self-face recognition advantage effect is not exist when partner face was processed. Keenan et al. (1999) showed that the right cortical hemisphere appears to be important for processing self-face. The effects of hand use (left or right hand), however, have not been taken into account. That's may be the reason why we failed to detect a significant interaction between self-esteem level and image types.

In our study, we have found that the pupil size of high group were larger when self-face, partner's face, and famous face were processing than unfamiliar face. In contrast, the pupil size of low self-esteem group was the same across the four types of images. A number of literature suggested that pupil size is an important indicator for eye movement tracking, and is reflected to a certain extent by mental activity (Zhang \& Ye, 2006). Moreover, some studies have demonstrated pupil size was positive relate to cognitive load, interesting and attitude (Han \& Yan, 2010; Partala \& Surakka, 2003). Thus, it can be explained that compare to the low self-esteem group, high self-esteem group show greater interest in all familiar images (self-face, partner's face, and famous face), and pay more attention to them.

In conclusion, we have demonstrated that 1) The processing is unique for individual to recognize self-face; 2) high selfesteem participants paid more attention to self-face, however, the low self-esteem participants paid more attention to others.

\section{Acknowledgements}

This study was supported by the Natural Science Foundation of China (Grant 30900399).

\section{REFERENCES}

Campbell, J. D., \& Lavallee, L. F. (1993). Who am I? The role of self-concept confusion in understanding the behavior of people with low self-esteem. In R. F. Baumeister (Ed.), Self-esteem: The puzzle of low self-regard. Plenum series in social/clinical psychology (pp. 3-20). New York: Plenum Press.

Creemers, D. H., Scholte, R. H., Engels, R. C., Prinstein, M. J., \& Wiers, R. W. (2012). Implicit and explicit self-esteem as concurrent predictors of suicidal ideation, depressive symptoms, and loneliness. Journal of behavior therapy and experimental psychiatry, 43, 638646. http://dx.doi.org/10.1016/j.jbtep.2011.09.006

Di Paula, A., \& Campbell, J. D. (2002). Self-esteem and persistence in the face of failure. Journal of personality and social psychology, 83, 711. http://dx.doi.org/10.1037/0022-3514.83.3.711

Duval, S., \& Wicklund, R. A. (1972). A theory of objective self-awareness (238 p). Oxford: Academic Press.

Han, Y. H., \& Yan, G. L. (2010). The application of the method of eye movement analysis in preschoolers' cognitive research. Psychological Science, 1, 191-193.

Keenan, J. P., McCutcheon, B., Freund, S., Gallup, G. G., Sanders, G., \& Pascual-Leone, A. (1999). Left hand advantage in a self-face recognition task. Neuropsychologia, 37, 1421-1425. http://dx.doi.org/10.1016/S0028-3932(99)00025-1

Kircher, T. T., Senior, C., Phillips, M. L., Rabe-Hesketh, S., Benson, P. J., Bullmore, E. T., \& David, A. S. (2001). Recognizing one's own face. Cognition, 78, B1-B15. http://dx.doi.org/10.1016/S0010-0277(00)00104-9

Partala, T., \& Surakka, V. (2003). Pupil size variation as an indication of affective processing. International journal of human-computer studies, 59, 185-198.

http://dx.doi.org/10.1016/S1071-5819(03)00017-X

Rosenberg, M. (1965). Society and the adolescent self-image. Princeton, NJ: Princeton University Press.

Seery, M. D., Blascovich, J., Weisbuch, M., \& Vick, S. B. (2004). The relationship between self-esteem level, self-esteem stability, and cardiovascular reactions to performance feedback. Journal of personality and social psychology, 87, 133. http://dx.doi.org/10.1037/0022-3514.87.1.133

Sui, J., Zhu, Y., \& Han, S. (2006). Self-face recognition in attended and unattended conditions: An event-related brain potential study. Neuroreport, $17,423-427$. http://dx.doi.org/10.1097/01.wnr.0000203357.65190.61

Tong, F., \& Nakayama, K. (1999). Robust representations for faces: Evidence from visual search. Journal of Experimental Psychology: Human Perception and Performance, 25, 1016. http://dx.doi.org/10.1037/0096-1523.25.4.1016

Wang, X. D., \& Wang, X. L. (1999). Rating scales for mental health. Beijing: Chinese Mental Health Journal Press.

Wang, Y., \& Luo, Y. J. (2005). Standardization and assessment of college students' facial expression of emotion. Chinese Journal of Clinical Psychology, 13, 396-398.

Zhang, X. F., \& Ye, W. L. (2006). Review of oculomotor measure since current reading research. Studies of Psychology and Behavior, 4, 236-240. 